\title{
Jüdische Buchgestalter der russischen Avantgarde
}

Den Ausgangspunkt für vorliegenden Beitrag bildet die Beschäftigung mit avantgardistischer Buchgestaltung, vor allem in der Tschechoslowakei, der in diesem Kontext eine als paradigmatisch anzusehende und ihrer Zeit europaweit anerkannte Vorreiterrolle zukommt, aber auch in Polen und Russland. Gerade im Hinblick auf russische avantgardistisch gestaltete Bücher fallen im Unterschied zu den tschechischen Büchern einige Besonderheiten auf (vgl. Hultsch 2019), die sich meiner Meinung nach nicht oder nicht nur darauf zurückführen lassen können, dass die historischen und kulturellen Ausgangsbedingungen für die jeweilige Avantgarde unterschiedlich waren und auf unterschiedliche nationale Traditionen zurückgegriffen werden konnte bzw. wurde.

Was die russische Avantgarde im Gegensatz zu der tschechischen aufweist, sind

a) eine neoprimitivistische Tendenz in ihrer Anfangsphase, also im frühen Futurismus;

b) allgemein eine verstärkte Hinwendung von einer stark auf das Wort konzentrierten (exklusiven Hoch-)Kultur zur Bildkultur und

c) eine Umschlaggestaltung, die eine nicht zu übersehende Tendenz aufweist, die Fläche des Umschlags und zum Teil auch der Seiten in mehrere Segmente zu gliedern und diese teilweise zu rahmen.

Die neoprimitivistische Tendenz (a), die sich darin niederschlägt, dass Texte mit Hand geschrieben werden, kann im Zusammenhang mit der Verfremdungstheorie Viktor Šklovskijs, die er in seinem Aufsatz Iskusstvo kak priem [Kunst als Verfahren] (1917) formuliert hat, erklärt werden. Es geht darum, den Prozess der Wahrnehmung zu verzögern, gewohnte Sehweisen aufzubrechen und Altvertrautes bewusst neu zu sehen (Šklovskij 1969 [1916], 14/15). Bei der manuellen Fertigung der Bücher setzt dieser Verzögerungsprozess nicht erst bei der Rezeption, sondern bereits bei der Herstellung der Texte ein. Das langsame Schreiben als Operation fördert die Kognition. Emotionen des Schreibers, die den Textinhalt um eine weitere Dimension erweitern, können in jedem Buchstaben zum Ausdruck kommen, der dadurch belebt wird.

Anne Hultsch, E-Mail: anne.hultsch@univie.ac.at 
Das von Aleksej Kručenych herausgegebene Buch Vzorval' [Explosion, Sprengung] (2. Aufl., [Sankt Peterburg]: Izd-vo Kuz'mina i Dolinskogo 1914) ist sowohl von Hand als auch mit Stempeln geschrieben, die lithographierten Illustrationen stammen von fünf verschiedenen Künstlern, wobei die letzte Seite die einzige von Natan Al'tman (1889-1970) gestaltete in diesem Buch ist (Kručenych 1914, [63]): ${ }^{1}$

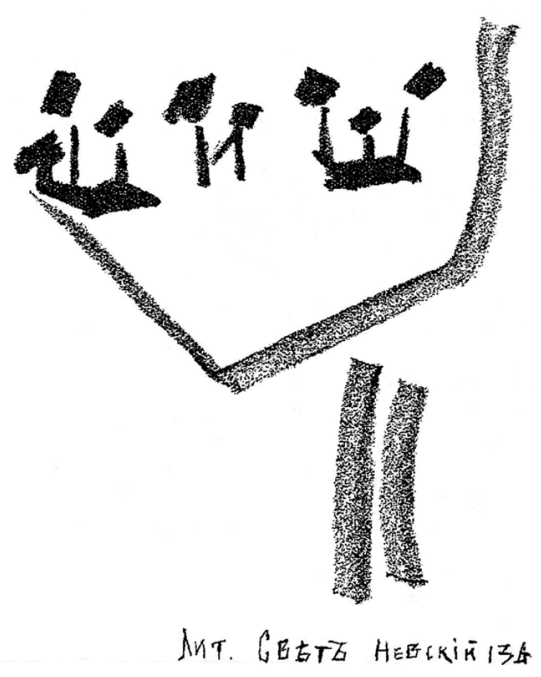

Abb. 1: Von Natan Al'tman gestaltete Seite in Aleksej Kručenych, Vzorval' (2. Aufl. 1914).

Der Künstler treibt mit dem Betrachter sein Spiel, indem er vortäuscht, dass es sich um hebräische Buchstaben handle (ש[?]). Es sind jedoch kyrillische, das heißt, das vermeintlich Hebräische erscheint nur als Bild, ${ }^{2}$ ergibt jedoch keinen

1 Al'tman schuf später auch Buchumschläge, sowohl für hebräisch als auch für kyrillisch geschriebene Bücher, wie z.B. 1922 für Got der fayer [Gott-Feuer] von Iechezkiel' Dobrušin und 1923 für Lik vojny [Antlitz des Krieges] von Il'ja Ėrenburg, wobei auffällig ist, dass bei beiden die Buchstaben zu Teilen der Graphik werden und ähnliche graphische Formen verwendet werden. Die hebräische Schrift bildet einen Halbkreis, die kyrillische ein ungeschlossenes Quadrat, die jeweilige Form wird dann durch Halbkreis und eckige Formen komplementiert.

2 Eine Interpretation sieht lt. Kacis (2006, o. P. - dort auch eine kolorierte Abbildung der Seite) in dem Bild, als Bild, einen achtarmigen Chanukka-Leuchter (dann wäre naheliegend, dass es sich bei dem mittleren Buchstaben um ein falsch geschriebenes $\boldsymbol{b}$ handeln würde), eine weitere die Bewegungen eines jüdischen Tanzes, der Freude und Trauer ausdrückt. Kacis selbst stellt eine Verbindung zu dem Bejlis-Ritualmordprozess her, in dem der Buchstabe $w$ eine wichtige Rolle gespielt habe. 
Text. Damit korrespondiert, dass einige Seiten zuvor die Seite diagonal geteilt ist, wobei auf der linken Hälfte als Text steht:

27 апреля в 3 часа пополудни / я мгновенно овладел / в совершенстве всеми / языками Таков / поэт современ/ности / Помещаю свои / стихи на / японском / испанском / и / еврейском / языках: (Kručenych 1914, [59])

[Am 27. April, um 3 Uhr nachmittags, beherrschte ich sofort alle Sprachen perfekt. So ist der Dichter der Gegenwart. Ich habe meine Gedichte auf Japanisch, Spanisch und Hebräisch verfasst:]

Auf der rechten Hälfte der Seite ist dann zu sehen, was der Dichter verfasst hat: einen kyrillisch geschriebenen Text in der transrationalen oder transmentalen Zaum'-Sprache der Futuristen. Das Japanische, Spanische und Hebräische wird so in den asemantischen Text eingeschrieben. Es ließe sich demzufolge die von Al'tman angefertigte hebräische Buchstabenfolge auch als - nun hebräisch geschriebener - Zaum'-Text auffassen. Liest man den Text jedoch als kyrillisch geschriebenen, ergibt sich das russische Palindrom „шиш“ (šiš) - es kann also wie das Hebräische auch von rechts nach links gelesen werden -, das ,Feige“, aber auch ,Feigenhand' heißt (ugs. für grobe Geste, wenn der Daumen zwischen Zeige- und Mittelfinger durchgesteckt wird) und in etwa bedeutet: Du hast von mir nichts zu erwarten; bekommst nichts von mir. Durch die Vortäuschung, dass es sich um hebräische Buchstaben handle, werde damit an die jiddische Redewendung „kiss mein toches“ [sinngemäß: ,Leck' mich am Arsch'] alludiert (Janecek 1984, 95-96, Anm. 18). Unten auf der Seite wird dann entschlüsselt, zu wem diese drastische Abgrenzung mit explosiver Kraft (vgl. den Buchtitel) stattfindet: „Лит. Свътъ. Невскій 13[?]“ [Lit. Licht. Nevskij 13[?]]. Wahrscheinlich ist 136 gemeint, denn an dieser Adresse (Nevskij-Prospekt 136) befand sich 1913 in St. Petersburg die Lithographische Werkstatt „Svet“ [Licht], so dass es sich also um die Einrichtung handeln dürfte, die das Buch hergestellt hat. Ob die gesamte Seite als hintergründige Reaktion auf einen Streit mit der Werkstatt, auf deren eventuell ablehnende Haltung gegenüber dem avantgardistischen Charakter des Buches oder nur gegenüber dem (pseudo-)hebräischen Charakter dieser einen Seite zu deuten ist, kann heute wohl nicht mehr geklärt werden. Wenn man das Buch auf den Kopf stellt, sehen die Buchstaben zudem aus wie Noten in einem Haus. Während hier also, erleichtert durch die inexakte Handschriftlichkeit, drei Notationssysteme gleichzeitig aufgerufen werden (können), was die Wahrnehmung verlangsamt, ging es der nachrevolutionären russischen und der westlichen Avantgarde hingegen eher um die Beschleunigung der Wahrnehmung und entindividualisierte (Pseudo-)Objektivität.

Den Schwerpunkt der Kultur von der Wort- auf die Bildkultur zu verlagern (b), hängt in Russland mit dem weit verbreiteten Analphabetentum zusammen, 
das kaum eine andere Möglichkeit zuließ, wenn man breitere Massen ansprechen wollte. Dazu dienten früher die Lubki (Volksbilderbögen), kurz nach der Revolution dann die sogenannten ROSTA-Fenster. ${ }^{3}$ Diese beiden Bild und Text verknüpfenden Medien stellten belehrende - moralisierende respektive agitierende Gebrauchstexte dar, während der reinen Schriftkultur Exklusivität zukam, weil sie nur einem kleinen Teil der Bevölkerung zugänglich war. Da die jüdische Kultur traditionell eher mit der Wort- oder Schrift- denn der Bildkultur verbunden wurde, heißt dieser Punkt für jüdische avantgardistische Buchgestalter, dass sie entweder auf die genannten russischen folkloristischen Erscheinungen zurückgreifen oder nach einer jüdischen Bildtradition suchen mussten, die sie weiter verarbeiten konnten.

Die aus meiner Sicht am schwierigsten zu klärende Frage ist die nach dem Grund für die andersartige Gestaltung der Buchumschläge (c). Und hier kommt nun das ins Spiel, was es berechtigt erscheinen lässt, dem Thema im Kontext von „Text und Bild in der jüdischen Literatur“ nachgehen zu wollen. Die These, die nun weiter verfolgt werden soll, lautet, dass eine Ursache für die Spezifika der russischen Avantgardeumschläge in der Prägung ihrer Autoren durch die jüdische Kultur zu sehen ist. Diese Idee wird nun in zwei Schritten verfolgt. Zunächst wird auf einige Beispiele aus dem Umfeld der Kul'tur-Liga eingegangen, um dann zu Beispielen überzugehen, die vordergründig keinen Einfluss der jüdischen Kultur mehr zeigen.

\section{Buchgestalter im Umfeld der Kul'tur-Liga}

Die Kul'tur-Liga wurde 1918 in Kiev, das nach der Februarrevolution 1917 zum Zentrum jiddischer Kultur geworden war, gegründet. Ihr programmatisches Ziel bestand darin, die Entwicklung der Kultur in jiddischer Sprache zu fördern (vgl. Kazovskij 2002, 17), wozu sie einen eigenen Verlag gründete, ein Buchhandelsnetz in der Provinz aufbaute, Kindergärten betrieb, die entsprechend mit neu herausgegebenen jiddischen Kinderbüchern versorgt wurden. Auch wenn sie in einzelnen Sektionen organisiert war, wurde ein ganzheitlicher Ansatz vertreten, der alle Bereiche des Lebens erfasste und auch dazu führte, dass nicht nur verschiedene politische Parteien unter diesem gemeinsamen Vorzeichen koalierten, sondern auch Literaten und bildende Künstler kooperierten (vgl. Wolitz 1995, 95; Kazovskij 2002, 23, 35). Das Anliegen der Kul'tur-Liga steht in engem Kontext

3 Auch Lisickij spricht davon, dass ihr Auditorium „velké množství poloanalfabetů - massa“ [die große Menge Halbanalphabeten - die Masse] gewesen sei (El Lissitzky 1929, 176). 
mit der sogenannten ,jüdischen Renaissance' in Europa, allerdings war ihr aufgrund der politischen Situation im ehemaligen Russischen Reich (ab 1921 dann der Sowjetunion) nur eine kurze Lebenszeit beschieden. Als 1917 der Erlass aufgehoben wurde, der das Publizieren in hebräischer Schrift verbot (Wolitz 1987, 41), belebte sich schnell die literarische Landschaft, deren Blüte gegen 1920 bis 1922 erreicht wurde. Bereits 1924 stellte die Kul'tur-Liga ihre Tätigkeit ein, der Verlag existierte noch bis 1930 (vgl. Kazovskij 2002, 27). So positiv diese kurze nachrevolutionäre Aufbruchsstimmung, die eine Vielzahl interessanter Bücher hervorbrachte, auch gesehen werden kann, so soll doch auch eine Kritik an der ganzen Bewegung nicht verschwiegen werden, die diese als Versuch der offiziellen Ideologie deutet, eine ausschließlich säkulare jüdische Kultur ohne Judaismus nur auf Grundlage des Jiddischen zu schaffen (Aržanuchin 2002, 202).

Den ersten Schritt für viele der Buchgestalter der Kul'tur-Liga bildeten ethnographische Expeditionen in das Gebiet der heutigen Ukraine und des heutigen Belarus zu den Quellen des osteuropäischen Judentums an den Ufern des Dnepr, an denen sie sich 1915/1916 im Auftrag der Jüdischen Historisch-Ethnographischen Gesellschaft beteiligten. Bei diesen lernten sie die jiddische Folklore, Symbole, die sie beispielsweise von Grabsteinen abzeichneten, Synagogenausmalungen usw. kennen. Aus diesem ethnographischen Interesse erwuchs ein ästhetisches. Sie fanden hier die Ornamente und dekorativen Elemente, auf denen sie ihre ersten eigenen Arbeiten basieren ließen - und ihnen damit bewusst einen Hauch von Fremdheit gegenüber der Kultur der Mehrheitsgesellschaft verliehen. Ein solches Interesse an archaischer Volkskunst pflegte auch die russische und die westeuropäische Avantgarde.

Direkt inspiriert von den Motiven der traditionellen Kunst ist Isachar-Ber Ribaks (1897-1935) Umschlag zu dem von Dovid Bergelson herausgegebenen Almanach der Literatengruppe Eygns [Eigenes] von 1920 (Kiev: Kultur lige). ${ }^{4}$ Das Buch enthält Dichtung und Prosatexte verschiedener Autoren. Auf dem Umschlag überlagern einander Schrift und Dekor, das hier aus einem tapetenstoffartigen Verwobensein von springenden/gehetzten Hirschen (Symbol für die Verfolgung der Juden) und Blumen besteht, so dass man weder Schrift noch die einzelnen Elemente des Dekors auf den ersten Blick wahrnimmt. Die Schrift wird zum Teil des Dekors, das Dekor zum Teil der Schrift. Die traditionellen jüdischen Motive werden durch die Aufschrift zum Eigenen erklärt. Es findet also nicht nur durch die Wahl traditioneller Motive, sondern auch auf Textebene ein Bekenntnis zur jüdischen Kultur statt.

4 Eine Abbildung des Umschlags ist zu finden unter: https://www.mahj.org/fr/decouvrir-collec tions-betsalel/eygns-48987 (3. September 2019). 
Ebenfalls wesentlich von der Expedition zehrte der Begleiter Ribaks: Lazar' (Eliezer) Markovič Lisickij (d. i. Ėl' Lisickij bzw. El Lissitzky; 1890-1941). Er gestaltete gleich nach ihrer Rückkehr nach Moskau das wohl bekannteste Buch der russischen jüdischen Renaissance, Sikhes-khulin [Plaudereien] von Moše Broderzon (Moyshe Broderson) (Moskva: Naše iskusstvo 1917), das auch unter dem Titel Prager Legende bekannt ist. Inhaltlich geht es um eine Plauderei mit einem Rabbi, die als mittelalterliche Legende aus Prag stilisiert wird (die Legende spielte eigentlich in Konstantinopel). Ein Rabbi dient am Hof einer Prinzessin, die er heiratet, um nicht sterben zu müssen. In einem Traum sieht er sich dann wieder bei seiner Familie als frommer Jude in Prag. Die Prinzessin gibt ihn gegen sein Versprechen, zu ihr zurückzukehren, für ein Jahr frei. Im Gegensatz zu ihm, der in Prag wieder ein angesehener Rabbi geworden ist, vergisst die Prinzessin sein Versprechen nicht, kommt nach Prag, küsst ihn mit seiner Erlaubnis ein letztes Mal. Durch diesen Kuss stirbt der Rabbi, ohne seine Religion und Frömmigkeit geändert zu haben.

Dieses Buch gilt laut Wolitz als „erstes säkulares jiddisches Kunstbuch“ (Wolitz 1995, 95). Auch hier tauchen in der Gestaltung bereits traditionelle Motive auf, deren Ursprung aber nicht nur in der Folklore zu suchen ist, sondern durch die Handkoloration zudem in mittelalterlichen hebräischen illuminierten Handschriften sowie der äußeren Form eines Teils der Auflage nach in Schriftrollen (Megilla) (Abramsky 1987, 62), die hier in einem sargartigen Kasten aus Eichenholz, der mit Brokat ausgeschlagen ist, liegen. Wie man den Abbildungen entnehmen kann, ${ }^{5}$ variieren die Verteilung und die Beziehung von Text und Bild von Seite zu Seite. Der Text wurde von einem professionellen Schreiber (Sofer) geschrieben, dessen Name im Kolophon durch drei Punkte angegeben wird. Nicht nur die Aufmachung des Buches, sondern auch sein Inhalt, der, wie dargestellt, das Sujet einer mittelalterlichen jüdischen Legende aufgreift, wirken anachronistisch, so dass beides gut miteinander korrespondiert und von einer engen Zusammenarbeit zwischen Autor und Künstler zeugt (Kazovskij 2002, 75).

Die aufwendige äußere Aufmachung und die Stilisierung beider Verfasser auf dem Titelblatt zu frommen chassidischen Juden (Kleidung, Bärte; unten: Autor, links: Künstler) generiert den Anspruch, dass es sich mit dem Buch a) um ein authentisches national-jiddisches Kunstwerk handle (Kazovskij 2002, 75) und b) die Kunst an die Stelle des Glaubens trete (Wolitz 1987, 29). Die Schrift wird so integriert, dass sie kaum noch als solche erkennbar ist. Sie wird zum Teil des

5 Einen Überblick über den Aufbau der einzelnen Seiten kann man aus dem kompletten Abdruck des Buches in: Kazovskij (2011, 66-80) gewinnen. Der Abdruck erfolgt dort in einer Größe, die es auch gut ermöglicht, den Text zu lesen. 
Bildes, wird Bild, was auf ihre ästhetischen Qualitäten verweist. Die Buchstaben übernehmen jenseits ihrer, eigentlichen' Funktion eine eigenständige graphische Rolle.

Dass ein Lisickij an der gesamten Arbeit interessierender Aspekt gerade die Form der Buchstaben war, kann man dem - merkwürdigerweise - zweisprachigen Kolophon entnehmen, der in der äußeren Form einem Thoraschrein nachgebildet ist. Lisickij definiert hier das Ziel seiner Illustrationen dahingehend, dass es ihm darum gegangen sei, den Stil und Inhalt der Ornamentzeichnung mit der wunderbaren assyrischen Schrift zu verbinden, ${ }^{6}$ es ist also weniger der Inhalt, der Text, der ihn zu seinen Illustrationen inspiriert hat, als vielmehr die Zeichen der Schrift, in der der Text geschrieben ist.

Aus der Auswahl von Arbeiten aus dem Kontext der Kul'tur-Liga sei abschließend noch eine Arbeit Mark Šagals (Chagall; 1887-1986) gezeigt, die ebenfalls relativ bekannt ist: der Umschlag bzw. die Titelseite zu Dovid Hofštejns (Hofshteyn) Gedichtzyklus Troyer [Trauer] (Kiev: Kultur lige 1922). ${ }^{7}$ Dieser scheint im gegebenen Kontext vor allem deshalb interessant, weil er durch die diagonale Schrift und die Konzentration auf die Schrift als gestalterisches Element für Chagall schon fast untypisch avantgardistisch wirkt. ${ }^{8}$ Der Leserichtung folgend ist die von Chagall entworfene Diagonale allerdings im Gegensatz zur - linken Avantgarde nicht aufsteigend optimistisch, sondern abfallend, wodurch er die Avantgarde mit ihren eigenen Mittel schlägt. Hier werden nicht die Buchstaben zu Bildern zusammengefügt, sondern die - bewusst nicht in Quadratschrift gesetzten - Buchstaben selbst, damit die Verbindung des Geschehens zum osteuropäischen Judentum hergestellt wird, dienen teilweise als Grundierung für

6 «В основу этой легенды взята схематическая заметка одной из пражских хроник. Задачей художника было слить в графических украшениях стиль и содержание с чудесным ассирийским письмом.» [Als Basis für diese Legende diente eine schematische Anmerkung aus einer Prager Chronik. Die Aufgabe des Künstlers bestand darin, in der graphischen Ausschmückung Stil und Inhalt mit der wunderbaren assyrischen Schrift zu verschmelzen.] (Broderson 1917, letzte Seite). Vgl. Apter-Gabriel 1987, 104.

7 Ausführlich zu dem gesamten Zyklus und den Illustrationen Wolitz 1995 und Koller 2009. Der Umschlag und alle Illustrationen sind zu finden unter: https://www.mahj.org/fr/decouvrir-collec tions-betsalel/troyer-52676 (9. September 2019).

8 Ähnlichen Prinzipien folgend, gestaltete er die Titelseite der Zeitschrift Shtrom (farbig abgebildet in Kazovskij 2011, 186): eine abfallende - fließende oder dahinströmende (vgl. den Zeitschriftentitel) - Leserichtung und die Buchstaben als Träger weiterer Informationen resp. belebte Bilder. Im ersten Buchstaben (w) ist ein schreitender Mann abgebildet (d. i. eventuell eine Anspielung an Chagall selbst [s. folgende Anmerkung]), das $า$ setzt sich aus einer Hand und einem Kopf zusammen; im o steht das Wort für ,Monat‘ (khoydesh), im ๖ das für ,Heft‘ (heftn), so dass das monatliche Erscheinen der Zeitschrift mitgeteilt wird. 
Bilder. ${ }^{9}$ In Troyer über die Pogrome während des Bürgerkriegs in der Ukraine (1919/20) werden gleich zu Beginn die Buchstaben des Namens Dovid in zwei Gewehre (2x Daleth, т) und eine Gewehrmündung oder Kugel (Waw, ו) verwandelt. Der Autor schafft mit seinen Texten Waffen. Der erste Buchstabe des Titels (Taw, ת) stellt gleichzeitig eine Axt, einen Schofar und ein Phylakterion dar. Das Resch ( $(7)$ ist eine Sense, in die ein weißer Fuchs gemalt ist, das nächste Waw (१) ist eine Axt, das letzte Resch ( 7 ) wurde von seiner Position her umgedreht und farblich invertiert, wodurch es einen Schofar darstellt, in den ein Haus, Pferd und Wagen hineingemalt sind (frei nach: Schwerter zu Pflugscharen). Es ließe sich noch weiter in die Details gehen, es sei aber nur noch darauf hingewiesen, dass hier die beiden Künstler (Hofštejn über Chagall) als Christusfiguren am orthodoxen Kreuz, dessen Diagonale umgedreht ist, dargestellt werden, die durch das Werkzeug der ukrainischen Bauern (Äxte, Sensen) an dieses genagelt sind (Wolitz 1995, 98, 100). Auch die Illustrationen im Text kombinieren die bildhaften Elemente mit Buchstaben, wodurch ihnen weitere Bedeutungen eingeschrieben werden.

Zum Vergleich, welche Gestalt ein vom Judentum des Autors beeinflusstes russischsprachiges Buch durch einen selbst nichtjüdischen Künstler erhalten kann, sei der Gedichtband Chevronskoe vino [Wein von Hebron] (Moskva: Vserossijskij sojuz poètov 1923) von Matvej Rojzman in der Gestaltung von Georgij Ečeistov (1897-1946) angeführt. ${ }^{10}$

9 Sein Selbstbildnis von 1918 führt dies mit kyrillischen Buchstaben vor, wo Chagall selbst zum л/l seines Namens wird, dessen wörtliche Bedeutung ,šagal‘ [eine männliche Person schritt] dargestellt wird (s. Koller 2014, 26-27).

10 Im Buch selbst wird Ečeistov fälschlich als „Egeistov“ angegeben (Rojzman 1923, 48), was mehr oder weniger auf einer Spiegelung von ч zu г beruht. Das Buch enthält Anmerkungen, in denen in den Gedichten auftretende kyrillisch geschriebene hebräische Worte und jüdische Realia erklärt werden (Rojzman 1923, 43-46). Es richtet sich also an ein Publikum, das mit diesen nicht vertraut ist. Rojzman übersetzte zu dieser Zeit aus dem Jiddischen, bewegte sich aber vor allem in russischen Dichterkreisen, vor allem dem der Imaginisten. 


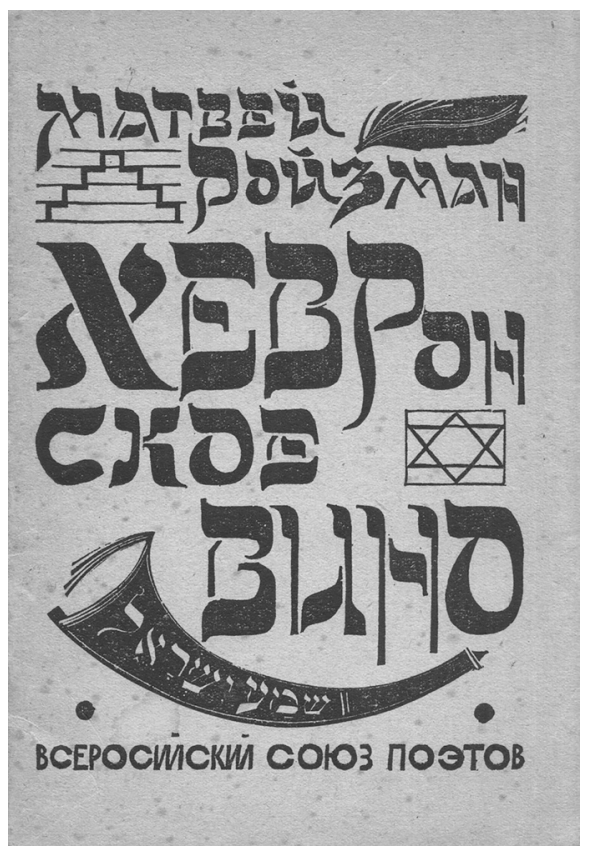

Abb. 2: Von Georgij Ečeistov gestalteter Umschlag zu Matvej Rojzman, Chevronskoe vino (1923).

Ečeistov ruft offensichtlich alles auf, was ihm zum Jüdischen einfällt: (pseudo-) hebräische und hebräische Schrift, eine stilisierte Klagemauer(?), Davidstern, Schofar etc. Die Inschrift des Schofars (shema yisrael) korrespondiert mit dem Bild und auch dem Text des ersten Gedichtes („Kol nidrej“), das sich um den Versöhnungstag dreht und an dessen Schluss dann auch das ganze Gebet in kyrillischen Lettern zitiert wird („Шма, Иероэл [...]“; Rojzman 1923, 16). Schofar und eingeschriebener Text sind am Versöhnungstag sowohl ideologisch als auch rituell miteinander als Glaubenssymbole verbunden. Es ist interessant, dass man, obwohl Ečeistov die kyrillische Schrift zum Teil aus hebräischen Buchstaben konstruiert, dennoch nicht in die Versuchung kommt, diese tatsächlich als hebräische Schrift wahrzunehmen. Die hebräischen Buchstaben werden in dem oberen Teil der Seite rein als Bauelemente für das Kyrillische verwendet, wodurch das Hebräische in das Kyrillische eingeschrieben wird. Die dadurch entstandene Schrift sieht fremd aus und es irritiert, dass Vokale ausgeschrieben sind bzw. dass der hebräische Konsonant Samech ( $($ ) aufgrund seiner äußeren Ähnlichkeit, der geschlossenen Gestalt als kyrillischer Vokal (o) ausgegeben wird, dennoch besteht im Gegensatz zu dem oben vorgestellten „šiš“ kein Zweifel daran, dass es sich um kyrillische Schrift handle, was vielleicht durch die letzte Zeile mit der Verlagsangabe ebenso unterstützt wird wie durch die Tatsache, dass die erste 
Zeile linksbündig angeordnet ist. Die Feder dürfte hier wohl eher als allgemeines Symbol, als Hinweis auf die im Buch enthaltene Dichtung, verwendet worden sein. Die Gestaltung ist nach außen hin sehr abgeschlossen, es bleibt nirgends Platz, um etwas hinzuzufügen. Die Schrift spielt als gestalterisches Element auf diesem Umschlag die entscheidende Rolle, aber es kommt hier nicht zu der sich gegenseitig kommentierenden Verbindung von Schrift, graphischen Elementen und Bild(ern), wie es bei den anderen bereits vorgestellten und noch vorzustellenden Beispielen der Fall ist.

\section{2 Übergang zum Konstruktivismus}

Lisickij konnte, und dafür ist er selbstverständlich bekannter, auch ganz anders als das angeführte Beispiel der Prager Legende zeigte. Als Übergang zu seinem rein konstruktivistischen Schaffen können zwei Werke von 1922 betrachtet werden. Das ist zum einen der Umschlag zu den 4 Teyashim [4 Ziegenböcken] (Warszawa: Tarbut 1922) von Ben Zion Raskin, der ihm zugeschrieben wird (nur der Umschlag, die Illustrationen besorgte er nicht; Nisbet 1988b, 285). ${ }^{11}$

Auf diesem Umschlag werden die hebräischen Buchstaben Teil einer rein graphischen Lösung, denn durch die Konfrontation mit anderen graphischen Elementen, die alle die Vierzahl ausdrücken (lateinische Ziffer, arabische Ziffer, Punkte auf der rechten Hälfte, Striche auf der linken), werden sie eben auch ,nur als graphische Elemente wahrnehmbar. Oben in der Mitte befindet sich ein roter Halbkreis mit eingefügtem Element. Man könnte dies als abstrahierte Hammer und Sichel deuten, man kann das Element aber auch als etwas gedrehtes Daleth (7) lesen, das wiederum den Zahlenwert, vier hat. Ähnlich kann auch die Umrahmung der römischen IV in der Mitte unten als im gleichen Winkel gedrehtes Mem (๖) gelesen werden, dem der Zahlenwert ,vierzig‘ zukommt. Es handelt sich zudem um eines der seltenen Beispiele, bei dem mit Vokalzeichen gearbeitet wird, die hier sogar noch durch ihre farbige Gestaltung hervorgehoben, dadurch jedoch weniger als Teil der andersfarbigen Schrift wahrnehmbar bleiben. Auch sie weisen also zusätzlich zu ihrer Funktion einen rein graphischen Wert auf.

Zum anderen ist es die Illustration zu der Erzählung „Šifs-Karta“ [Schiffsticket] aus den Šest' povestej o legkich koncach [Sechs Erzählungen mit leichtem Ende] von Il'ja Ėrenburg (Moskva/Berlin: Gelikon [Helikon] 1922).

11 Eine Abbildung ist $\mathrm{zu}$ finden unter: https://www.moma.org/collection/works/7542?loca le=en\# (9. September 2019). 


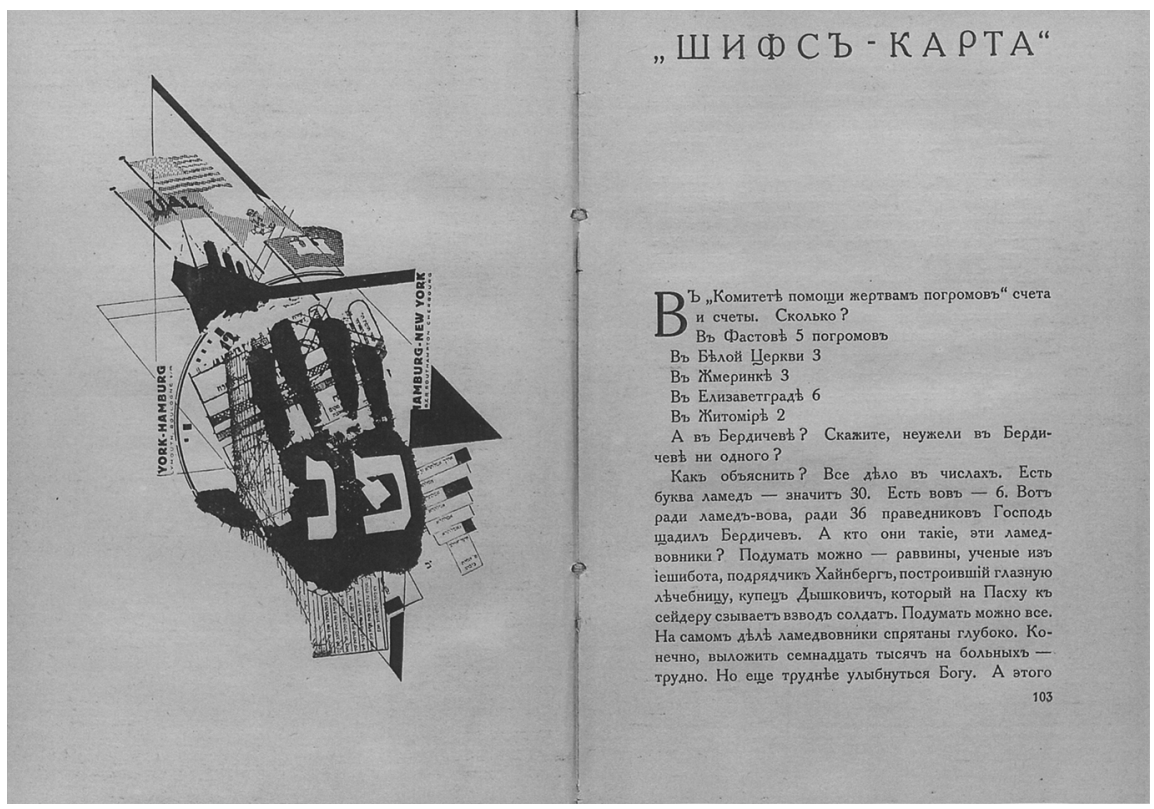

Abb. 3: Von Lazar' Lisickij gestalte Illustration und Textseite in Il'ja Ėrenburg, Šest' povestej o legkich koncach (1922).

In diesem Fall handelt es sich um ein russisches Buch, wobei auf der montierten Illustration nur lateinische und hebräische Schrift zu sehen ist, jedoch keine kyrillische, die dem Text auf der Nebenseite vorbehalten bleibt. ${ }^{12}$ Dem entspricht der dem Protagonisten unverständliche Titel und Hauptgegenstand der Erzählung. Sein Sohn, der bereits nach Amerika emigriert ist, verspricht ihm die Zusendung einer „šifs-karta“. Da der Vater das „чужое и таинственное слово“ [fremde und geheimnisvolle Wort] (Ėrenburg 1922, 104) nicht versteht, assoziiert er es mit dem heilbringenden Messias, dessen Kommen ihm der Sohn versichert. Der Protagonist stirbt am Ende, obgleich er im Besitz eines Schiffstickets ist, im Pogrom.

Die Grundform der Abbildung bildet ein Davidstern, der Bewegung in das Bild bringt. Bei dem untergelegten Text handelt es sich um einen Ausschnitt aus der Mischna. Auf der Hand befinden sich die beiden Buchstaben Pe (פ) und Nun (), das Akronym für „po nikbar“ [Hier liegt], wie man es auf jüdischen Grab-

12 Interessanterweise folgt dieser der bis Mai 1917 gültigen Orthographie, was also gar nicht avantgardistisch ist und russischen Exilverlagen nur bei Büchern möglich war, die nicht für den Sowjetmarkt bestimmt waren (Janecek 1984, 18). 
steinen findet. Die Buchstaben erscheinen als Linien auf der Hand - es ist das Schicksal eines jeden, einst auf dem Friedhof zu liegen (dass diese Aussage leider von der Geschichte widerlegt werden sollte, wusste Lisickij noch nicht). Die Schichtung des Materials, dessen oberste Ebene die Grabinschrift bildet, die sich wiederum auf alles unter ihr liegende, also die jüdische Kultur, beziehen kann, macht diese Illustration nicht nur ästhetisch, sondern auch inhaltlich sehr eindrucksvoll. ${ }^{13}$ Wie der Text so verbindet auch die Illustration den Bereich des Religiösen mit dem des Weltlichen. Das eine erklärt das andere, oder stellt es in Frage.

Der daneben abgedruckte Text Ėrenburgs, der Anfang der Erzählung, beginnt mit der Aufzählung von Pogromen in verschiedenen Orten und der Verwunderung darüber, dass in Berdičev (bisher) keiner stattgefunden habe. Alles liege in den Zahlen begründet: „Есть буква ламед - значит 30. Есть вов - 6. Вот ради ламед-вова, ради 36 праведников Господь щадил Бердичев“ [Es gibt den Buchstaben Lamed - er bedeutet 30. Es gibt Waw - 6. Für Lamed-Waw, für 36 Gerechte hat der Herr Berdičev verschont] (Ėrenburg 1922, 103). Im weiteren Verlauf ist dann von den Lamedvovniki [Lamedwawnikern] die Rede, das heißt, die zwei einzelnen Buchstaben resp. Zahlen werden personifiziert. Aus ihnen werden also nicht nur Worte oder Bilder gebildet, sondern in diesem Falle werden sie sogar zu Personen. Wenn man den weiteren Weg Lisickijs in den Blick nimmt, dessen Illustration zu „Šifs-Karta“ als seine letzte gilt, in der Elemente des Hebräischen resp. Jüdischen Verwendung finden, könnte man zugespitzt sagen, dass diese Elemente auf ihn und seine Arbeiten übergehen und deshalb nicht mehr vordergründig gezeigt werden müssen. ${ }^{14}$

\section{Konstruktivistische Seitenarchitektur}

Im Hinblick auf die Entwicklung von Lisickij wird oft von einem Bruch oder einem kompletten Wandel gesprochen, als er sich von der jüdischen Thematik ab- und dem Konstruktivismus zuwandte. ${ }^{15}$ Außer den gezeigten Übergängen scheint es

13 Sabine Koller $(2014,20)$ schreibt, dass „Lisickij in der Illustration zu Il’ja Ėrenburgs Erzählung Šifskarta [...] das Jüdische und die mit ihm verbundenen messianischen Hoffnungen“ begrabe.

14 Er signiert sein bekanntes Selbstporträt (Der Konstrukteur) von 1924 mit „el“ und verwendet dann das Pseudonym „El Lissitzky“ (vgl. Koller 2014, 22), so dass auf diese Weise das Hebräische fortan jeder seiner Arbeiten, die er so signiert, eingeschrieben ist.

15 Peter Nisbet $(1988,13,20)$ spricht von einem „Übertritt“, einer „Konversion“ Lisickijs, die dieser selbst als „radikalen Bruch mit seiner künstlerischen Vergangenheit“ gesehen habe. Leider führt Nisbet allerdings keinerlei Belege für diese Selbstsicht Lisickijs an. Ähnlich Christiane 
mir jedoch eher so, dass er in struktureller Hinsicht auch in rein konstruktivistischen Werken an seine Auseinandersetzung mit der jüdischen Kultur anknüpfte. Buchseiten können auch als Bilder betrachtet werden, wie Paul Valéry 1926 formulierte:

Neben der Lektüre selber und unabhängig von ihr gibt es noch den bleibenden Gesamtaspekt alles Geschriebenen und Gedruckten. Eine Seite ist ein Bild. Sie liefert einen Totaleindruck, bietet dem Auge ein Ganzes oder ein Gefüge von größeren Blöcken und Schichten, von schwarzen Flächen und weißen Leerräumen, einen Fleck von mehr oder minder glücklicher Gestalt und Überzeugungskraft. Diese zweite Art zu sehen, nicht mehr sukzessiv und linear fortschreitend wie bei der Lektüre, sondern als eine Zusammenschau auf den ersten Blick, gestattet uns, die Typographie in die Nähe der Architektur zu rücken. (Valéry 1995 [1926], 467-468)

Für solch eine Seitenarchitektur, wo verschiedene Textblöcke und die Lücken zwischen diesen sowie verschiedene Schriften semantische Signifikanz erhalten, scheint gerade der Talmud das beste Beispiel zu sein, der unter dieser Prämisse eben nicht nur als ein Text-, sondern auch als ein Bildbuch betrachtet werden kann, dessen Flächen sich gegenseitig Sinn geben. Auch Nikolaj Burljuk (18901920) schreibt in dem Manifest Poètičeskie načala [Poetische Prinzipien] (1914), nachdem er die Bedeutung der jeweils konkreten (Hand-)Schrift hervorgehoben hat: „Громадное значение имеет расположение написанного на бумажном поле“ [Gewaltige Bedeutung hat die Anordnung des Geschriebenen auf der Fläche des Papiers] (N. Burljuk 2000 [1914], 150).

Um zu veranschaulichen, was damit gemeint ist, sei ein kurzer Zeitsprung in die Gegenwart gestattet, denn Joseph Sassoon Semah (geb. 1948), der sich theoretisch intensiv mit Chagall, Lisickij, Malevič, Mondrian und anderen auseinandergesetzt hat (vgl. Semah 2006), führt genau dies mit seinen Übermalungen des Talmud Bavely bestens vor Augen:

Schütz (1989, 148-149) oder Chimen Abramsky (1966, 182), der die jiddischen Bücher der Jahre 1917-1922 als ,isolated from the rest of his artistic achievement“ sieht. Beides (Jüdisches oder Hebräisches und Abstraktes oder Konstruktivistisches) zusammen zu denken, darum bemühen sich ausdrücklich Alan Birnholz (1973) und Igor Dukhan (2007, 61, 64): "If in 1916-19 El Lissitzky was, metaphorically speaking, predominantly 'Jewish,' between 1919 and 1921 primordially 'universal' (with hidden traces of Jewish time-sensitivity), in Berlin and Germany [1921-25] we could find Lissitzky 'Jewish' and 'universal,' or 'Jewish' as ‘universal,' with emphasis upon 'universal.”; "The non-ohjective and non-figural character of the synagogue space coincided with the nonobjectivity of the Suprematist vision. The avant-garde ideal had paradoxically discovered its reflection in the sacral Jewish space”. Leonid Kacis (2000 und 2005) untersucht den Zusammenhang von Suprematismus und jüdischem messianischem resp. apokalyptischem Denken. 


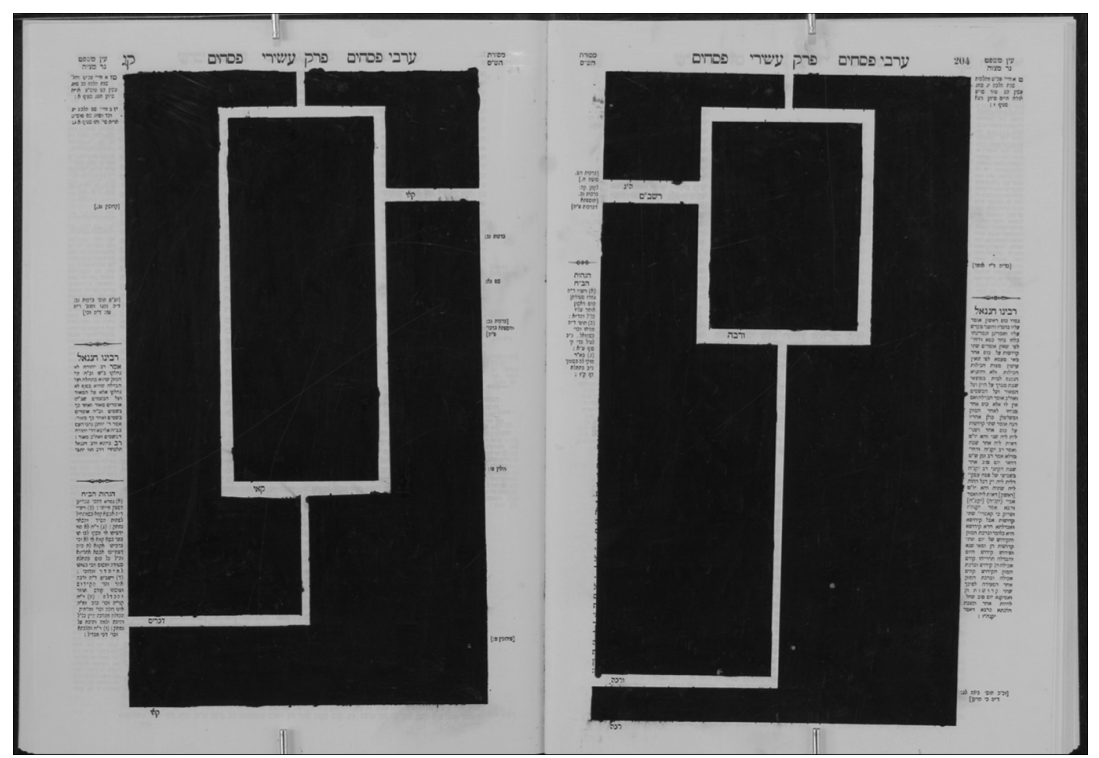

Abb. 4: Joseph Semah, An introduction to the principle of relative expression (1979).

Hier wird ein Detail von An introduction to the principle of relative expression (1979) gezeigt, aus denen Semah eine ganze Serie gebildet hat, um auf die Vielfalt hinzuweisen. Als Differenz zwischen der jüdischen und der christlich-westlichen Tradition sieht er 1.) die Asymmetrie des Aufbaus der Talmudseiten, die 2.) Ausdruck der fundamentalen Offenheit des jüdischen Denkens sei, so dass alle Positionen gegenüber der Tradition gleichwertig auf der Seite existieren dürfen. Es sind also zwei Begriffe, mit denen er hinsichtlich der jüdischen Typographie operiert: der der ,asymmetrischen Typographie“ und der der ,toleranten Typographie‘ (Hartog 2009, 18; vgl. auch Gilbert 2012), was auch bedeutet, der der ,unabgeschlossenen Typographie‘, denn:

Every word and every letter can have more than one meaning; all that the text in all generations has ever brought about in the life of the individual or of the community, makes up the content of that text. In the words of Rabbi Efraim of Sadylkov (second half $18^{\text {th }}$ century): 'The Torah is only half a book; through interpretating it will become a full book' (Aschkenasy 1994, 73)

In diesem Zusammenhang sieht man, das nur als Randbemerkung, dass der Einfluss der Lubki auf den Seitenaufbau russischer Avantgarde-Bücher geringer scheint, als zum Teil angenommen wird.

Diese erzählen (äußerlich Comics ähnlich, mehr Bild als Text) kurze belehrende Geschichten und zeigen drastisch die Folgen von - meist sozialem - Fehl- 
verhalten. Deshalb sind sie hierarchisch strukturiert. Als ein typisches Beispiel sei hier ein Lubok aus dem Jahre 1882 erwähnt, der vor zu viel Alkoholkonsum warnt. Der Titel lautet P’janstvo i ego užasnye sledstvija [Trunksucht und ihre furchtbaren Folgen]. Die symmetrische Anordnung zeigt mittig die Hölle, in die alles mündet. Die Warnung vor ihr bildet den Kern der Aussage, der keiner weiteren Deutung bedarf bzw. eine solche auch nicht zulässt. Diese Bild-Texte sind abgeschlossen oder, mit den Worten Semahs, intolerant gegenüber weiteren Interpretationen.

Auch die zweite mögliche Inspirationsquelle, russische Ikonen, die - wie z.B. viele Arbeiten Chagalls - gegen die westlichen Gesetze der Perspektive verstoßen (vgl. Friedman 1978), tritt in den Hintergrund, wenn man bedenkt, dass diese fast immer symmetrisch angeordnet sind und ganz klaren Gestaltungsvorgaben folgen, d.h. ebenfalls nicht gerade sehr offen für weitere Interpretationen sind.

Wenn man die späteren Arbeiten Lisickijs unter den Vorzeichen der Asymmetrie und der Toleranz des Seitenaufbaus des Talmuds betrachtet, dann scheint dies einen neuen Blick auf sein Werk zu ermöglichen. Nimmt man beispielsweise seine Gestaltung von Vladimir Majakovskijs Dlja golosa [Für die Stimme] (Moskva/Berlin: Gosudarstvennoe izdatel'stvo R.S.F.S.R. [Staatsverlag] 1923), dann zeigt sich die rein typographische Lösung (es wird bewusst das Wort Illustration vermieden, Lisickij wird eingangs als der ,Konstrukteur' genannt ${ }^{16}$ ) des gesamten Buches als Kommentar zu dem Text Majakovskijs.

16 In Šest’ povestej o legkich koncach war noch die Rede von den „risunki“ [Zeichnungen] Lisickijs. 


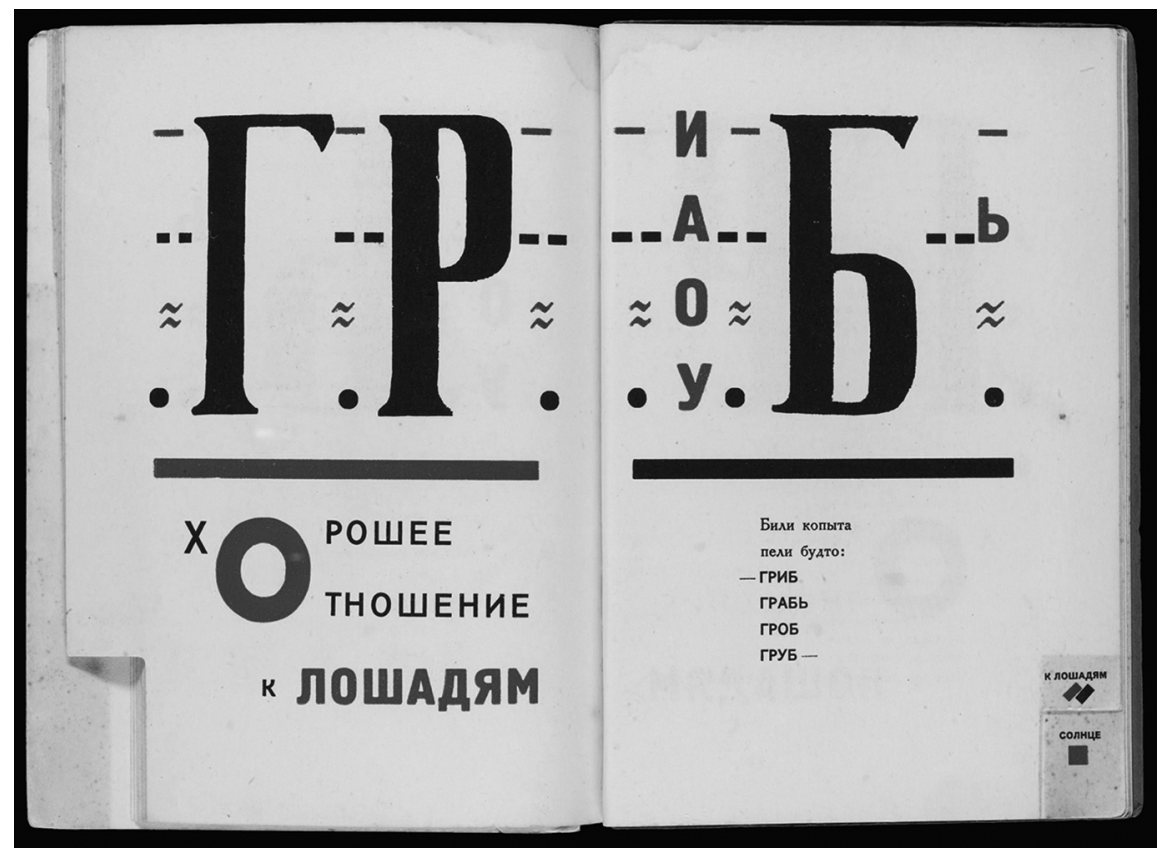

Abb. 5: Von Lazar' Lisickij gestaltete Doppelseite in Vladimir Majakovskij, Dlja golosa (1923).

Besonders interessant ist, was auf dieser Seite, dem Beginn des Gedichtes „Chorošee otnošenie k lošadjam“ [Gute Beziehung zu Pferden] zu sehen ist. Im oberen Teil verwandelt Lisickij das Kyrillische in eine Konsonantenschrift, indem er die Austauschbarkeit der rot geschriebenen Vokale andeutet. ${ }^{17}$ Bei gleichbleibendem Konsonantenbestand ( $\mathrm{C} / \mathrm{G}, \mathrm{P} / \mathrm{R}, \mathrm{B} / \mathrm{B})$ werden durch die Verwendung unterschiedlicher Vokale unterschiedliche Bedeutungen erzielt. Wie die Mundöffnung bei der jeweiligen Aussprache je nach Artikulationsort des Vokals aussieht (i: vorne, a: hinten, o: oben, $u$ : unten), zeigen die graphischen Zeichen: „grib“ [Pilz] - schmaler Mund (-); „grab”“ [raube, plünder] - Mund weniger schmal (- -); „grob“ [Sarg] - langsamer Übergang zum runden Mund ( $\approx)$;,,grub“ [grob] - runder Mund $(\bullet)$. Es bleibt dem Interpreten überlassen, welchen Vokal er einsetzt. Wenn man auf dieser Doppelseite zudem die groß und fett geschriebenen Buchstaben als Flächen betrachtet, ist man optisch nicht weit von Semahs Übermalungen entfernt, was ebenfalls für die nach außen hin offene Gestaltung der Seite gilt.

17 Die russische literarische Avantgarde vertrat die Vorstellung, dass vor allem Konsonanten sinnliches Empfinden auslösen (vgl. D. Burljuk 2000 [1913], 148). 
Das Kinderbuch Suprematičeskij skaz pro dva kvadrata v 6-ti postrojkach [Suprematistisches Märchen für zwei Quadrate in sechs Aufführungen] (Berlin: Skify [Skythen] 1922) ${ }^{18}$ beginnt gleich mit einer Provokation, nämlich mit der Aufforderung, nicht zu lesen, sondern sich die Welt aktiv anzueignen. Und so stehen denn Text(e) und verschiedene geometrische Elemente, die sich gegenseitig kommentieren auf einer Seite, um betrachtet zu werden. Der Blick wandert zwischen den verschiedenen Ebenen hin und her. Die gemeinsam mit der Aufforderung, nicht zu lesen, vorgebrachte Aufforderung, Papier, Griffel und ein Holzstück zu nehmen und selbst zu komponieren, zu malen und zu bauen, macht deutlich, dass es eben um diesen produktiven Prozess geht, der den bereits existierenden Kommentaren einen weiteren hinzufügen soll.

Viele der Seiten zeigen in einem (meist) quadratischen Rahmen große geometrische Figuren (Kreise, Quadrate) und in einigen Fällen auch Text, die stets anders angeordnet sind. Über diese topologischen Transfigurationen schreibt Igor Dukhan:

The montage visual structure of $O f$ Two Squares is based on a dramaturgy of topological transfigurations. Every new page puts forward a thrust of visual energy. The geometric bodies meet in the gravity fields, compressing and clashing with each other. The gravitation and energies of the pictorial field transform their geometries. This clash between forces of gravity and inertia leads to the compression and disappearance of bodies. The narrative sequence of discourse dissolves in the dynamic struggle of forces, gravity, and inertia. Montage of visual events, the ecstatic temporality of "folding" (the term of Gilles Deleuze) creates the dynamic visuality of a time stream. The ecstasies of time emerge as a fundamental force for construction of $O f$ Two Squares and form, as we will attempt to show later, an expressive trace of Jewish timesensitivity. (Dukhan 2007, 61)

Außerhalb dieses Rahmens befindet sich Schrift, die eben so dynamisch angeordnet ist und sich nicht linear lesen lässt. Diese ist mit geometrischen Formen (Linien) kombiniert, einige Buchstaben werden durch größere und/oder dickere Schreibung hervorgehoben. Die so gestaltete Schrift kommentiert oder verbalisiert, was in dem Rahmen zu sehen ist, bildet aber gleichzeitig durch ihre eigene Anordnung ein Buchstaben-Bild, das selbst zu einer Aussage wird. In Anlehnung an Semahs Skulpturen als Fußnoten zu seinen Texten, können Lisickijs Texte als Fußnoten zu seinen Prounen verstanden werden. Wir haben es hier also mit einer ähnlichen äußeren Struktur wie beim Talmud zu tun: eine zentrale Aussage, um die asymmetrisch verschiedene Deutungen gruppiert werden, was alles zusammen zu einer weiteren eigenen Deutung anregen soll. Die zentrale Aussage ist bei

18 Das gesamte Buch ist zu finden unter: https://www.moma.org/collection/works/11113?artist_i $\mathrm{d}=3569$ \&locale $=$ en\&page=1\&sov_referrer=artist (9. September 2019). 
Lisickij die bildliche, die durch Text außerhalb des Rahmens kommentiert wird. So ist es auch konsequent, dass der Text der letzten Seite lautet: „тут кончено дальше“ [hier beendet - weiter]. Das bringt Peter Nisbet dazu, von einer „Ouvertüre zu weiteren Entwicklungen“ und einem „Katalysator für eigene Aktivitäten des Lesers“ zu sprechen und fortzufahren:

In dieser Konzentration auf die Anfänge ist dieses Buch typisch für Lissitzkys Typografie. In den gesetzten, aber oft frei arrangierten Wörtern seiner vielen Layouts hob er den ersten Buchstaben, die Initiale, die für das ganze Wort stehen konnte, regelmäßig ganz besonders hervor. [...] Darüber hinaus erfüllten Buchstaben manchmal eine zweifache Aufgabe, das heißt, ein Schriftzeichen diente gleichzeitig zwei Wörtern, in denen es vorkam [...]. (Nisbet 1988a, 17)

In seinem für die tschechische Zeitschrift Typografia 1929 verfassten Beitrag „Kniha z hlediska zrakového dojmu - kniha visuelní“ [Das Buch aus dem Blickwinkel des Seheindrucks - das visuelle Buch] unterscheidet Lisickij zwischen Sprache und Schrift und führt aus, dass die dargestellte, visuelle Sprache reicher als die gesprochene sei. Diesen Vorteil habe das aus Buchstaben gesetzte Buch verloren, weshalb er annehme, dass die nächste Form dem plastisch-dargestellten Buch gehören werde, das mit dem Wort die Vorstellung abbilde (El Lissitzky 1929, 175). ${ }^{19}$ In diesem Beitrag wird der Titel des Quadrate-Buches mit $O$ dvou mocninách [Von zwei Potenzen] angegeben (El Lissitzky 1929, 178). ${ }^{20}$ Das scheint insofern interessant, weil es wiederum viele Interpretationsmöglichkeiten eröffnet: Sind damit nur die zwei Quadrate als widerstreitende Kräfte gemeint? Lisickij schreibt dazu: „Die Worte bewegen sich in den Kraftfeldern der handelnden Figuren: Quadraten. Die allgemeinen und plastischen Momente sollen hier typographisch gestaltet werden“ (El Lissitzky 1925, 152). Oder bezieht sich dieser Titel auch auf die beiden Größen Bild und Text/Schrift, die sich in „Kraftfeldern“ befinden?

\section{Fazit}

In der russischen Avantgarde kommt es zu einer engen Zusammenarbeit von Dichtern und Malern, so dass fast kein Gedichtband ohne Beteiligung eines Malers entstanden ist (El Lissitzky 1929, 176). Bücher sind massenhaft reproduzierbar und ermöglichen es so den modernen Künstlern, eine wesentlich brei-

19 Wenn der Text in Typografia auch als für die Zeitschrift geschriebener Originalbeitrag ausgegeben wird, ist er doch weitgehend identisch mit Lisickijs Text „Unser Buch“ (1926/27).

20 An dieser Stelle weicht die tschechische von der deutschen Version ab. 
tere Masse zu erreichen, als wenn sie ihre Arbeiten nur in Museen ausstellten. $\mathrm{Zu}$ der Ablösung der Kirchen, Paläste, Museen als Vermittlungsorte von Kunst durch Bücher äußerte sich bereits 1919 Lisickij:

\begin{abstract}
А ведь книга сейчас все. Она стала в наше время тем, чем некогда был храм, с его фресками и витражами (цветными окнами), чем были дворцы и музеи, куда люди приходили смотреть и познавать. Книга стала монументом современности, но в отличие от старого монументального искусства она сама идет в народ, а не стоит как храм на месте в ожидании, пока к нему не придут. И вот книга ждет от современного художника, чтобы он создал из нея этот монумент будущего. (Lisickij [1919])

[Und so ist das Buch jetzt alles. Es wurde in unserer Zeit das, was einst die Kirche war, mit ihren Fresken und Vitragen (farbigen Fenstern), was die Paläste und Museen waren, zu denen die Menschen zum Schauen und Erkennen kamen. Das Buch wurde zum Monument der Gegenwart, doch im Unterschied zur alten Monumentalkunst geht es selbst zum Volk und steht nicht wie die Kirche wartend an seinem Platz, bis das Volk zu ihm kommt. Und so erwartet das Buch vom zeitgenössischen Künstler, dass er aus ihm dieses Monument der Zukunft schafft.]
\end{abstract}

Die Exklusivität fällt mit der Abkehr von den per Hand hergestellten Büchern des frühen Futurismus weg. Das Streben nach Geschwindigkeit, das die westliche Avantgarde dazu gebracht hat, unter größtmöglichem Einsatz verschiedener technischer Mittel gleichzeitiges, in gewisser Weise auch standardisiertes Sehen möglich zu machen, steht einem Zweig der russischen Avantgarde gegenüber, der es gerade um die Kombination verschiedener struktureller Ebenen des Textes geht, deren Betrachtung langsamer erfolgen muss, weil es sich um je eigene Interpretationen handelt, die einander kommentieren. Einzelnen Buchseiten graphische Sorgfalt zu widmen, geht hier einerseits mit der Achtung gegenüber dem Wort einher und kann andererseits vielleicht auch dadurch begründet werden, dass es keine jüdische Monumentalkunst gibt und die Buchgestalter deshalb ihre Anregungen aus der jüdischen Volkskunst schöpften, also vor allem aus - zweidimensionalen - Grafiken (vgl. Aronson 1924, 52, 55).

Dem Einsatz von Buchstaben, mithin Text, bei der graphischen Lösung (die nicht einfach als Ornament dienen) kommt gerade bei jüdischen Buchgestaltern eine auffallend große Bedeutung $\mathrm{zu}^{21}{ }^{21}$ die vermuten lässt, dass auch in ,bilderlosen Zeiten' der graphische Eigenwert sowohl der einzelnen Buchstaben, die geometrisch einfache Formen aufweisen, als auch der Anordnung der Textblöcke auf der Seite klar erkannt worden ist:

21 "Some of the artists of the Kultur-Lige saw Hebrew font as the main and sometimes the only decorative element in constructing book illustrations. [...] Emphasizing the geometrical character of Jewish letters and combining them with the simplest geometrical lines and figures, Altman created font compositions that were 'read' as a visual image of the book title” (Kazovskij 2011, 14). 
Еврейская буква сама по себе уже элемент графический, всюду сохраняющий свою характерность: геометрическую простоту, замкнутость, цельность и необычайную способность связываться с соседней, образуя цельную, вязкую арабеску строки. [...] [O]ставшееся между строчками и буквами пространство бумаги играет не меньшую роль, чем покрытый чернилами рисунок. (Aronson 1924, 47, 51)

[Der hebräische Buchstabe ist für sich selbst bereits ein graphisches Element, das überall seine Spezifik beibehält: geometrische Einfachheit, Abgeschlossenheit, Totalität und die außergewöhnliche Fähigkeit, mit dem benachbarten Buchstaben in Verbindung zu treten und eine ganzheitliche, zusammenhängende Arabeske der Zeile zu bilden. [...] Der Abstand zwischen den Zeilen und den Buchstaben spielt keine geringere Rolle als die mit Tinte bedeckte Zeichnung.]

Es ist zu vermuten, dass dieses Bewusstsein für die Form der einzelnen Buchstaben und der Fläche seine Grundlage darin hat, dass es in der jüdischen Kunst nie um das naturalistische (mimetische) Abbilden ging. Es kann so der Wert der einzelnen Buchstaben zum Tragen kommen, die laut der Geschichte von Rabbi Chanina ben Teradion einzeln nach oben geflogen - und deshalb nicht samt dem Pergament der Thora-Rolle verbrannt sind. ${ }^{22}$ Die Buchstaben überleben und können/müssen von den Nachfahren wieder eingesammelt und (neu) angeordnet werden. Es scheint kein Zufall zu sein, dass in der Avantgarde dem Verwenden verschiedener Schrifttypen sowie von Handschrift auf einem Buchumschlag oder einer Buchseite programmatischer Charakter zukam. Die jüdischen Buchgestalter der russischen Avantgarde stellten Text und Bild nicht einander dichotomisch gegenüber, sondern verwandelten einerseits Buchstaben und Texte in (Teile von) Bilder(n) und nahmen andererseits Textseiten in ihrer Bildhaftigkeit wahr.

\section{Abbildungen}

Abb. 1: Von Natan Al'tman gestaltete Seite in Aleksej Kručenych, Vzorval' (2. Aufl. 1914, S. [63]), übernommen aus Russian Avant-garde Online. Advisor A. Krusanov. Leiden and Boston: Brill, 2004; http://primarysources.brillonline.com/browse/russian-avantgarde-19041946 (3. September 2019).

Abb. 2: Von Georgij Ečeistov gestalteter Umschlag zu Matvej Rojzman, Chevronskoe vino (1923).

Abb. 3: Von Lazar' Lisickij gestalte Illustration und Textseite in Il'ja Ėrenburg, Šest' povestej o legkich koncach (1922), S. 102/103.

Abb. 4: Joseph Sassoon Semah, An introduction to the principle of relative expression, 1979.

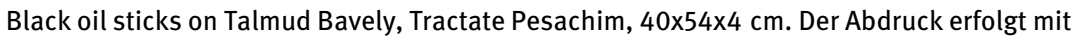
freundlicher Genehmigung des Künstlers.

22 Auf diese Geschichte ('Ab. Zarah 17b et seq.) rekurriert auch Semah, wie Aschkenasy (1994, 77, $85,93)$ darstellt. 
Abb. 5: Von Lazar' Lisickij gestaltete Doppelseite in Vladimir Majakovskij, Dlja golosa (1923), S. [52/53].

\section{Literatur}

Abramsky, Chimen. „El Lissitzky as Jewish Illustrator and Typographer“. Studio International 172.882 (October 1966): 182-185.

Abramsky, Chimen. „Yiddish Book Illustrations in Russia: 1916-1923“. Tradition and Revolution. The Jewish Renaissance in Russian Avant-Garde Art 1912-1928. Hg. Ruth Apter-Gabriel. Jerusalem: Israel Museum, 1987. 61-70.

Apter-Gabriel, Ruth. „El Lissitzky’s Jewish Works“.Tradition and Revolution. The Jewish Renaissance in Russian Avant-Garde Art 1912-1928. Hg Ruth Apter-Gabriel. Jerusalem: Israel Museum, 1987. 101-124.

Aronson, Boris. Sovremennaja evrejskaja grafika. Berlin: Petropolis, 1924.

Aržanuchin, Vladislav A. „Judaismus“. Lexikon der russischen Kultur. Hg. Norbert Franz. Darmstadt: WBG, 2002. 201-202.

Aschkenasy, Yehuda. „Typografie van tolerantie / Typography of tolerance“. Joseph Semah. Driedimensionale typografie. Amsterdam: Stichting Makkom, 1994. 67-95.

Birnholz, Alan C. „El Lissitzky and the Jewish Tradition“. Studio International 186.959 (October 1973): 130-136.

Broderson, Moyshe. Sikhes-khulin (Prager Legende). Moskva: Naše iskusstvo, 1917.

Burljuk, David et al. „,Sadok sudej““ [1913]. Literaturnye manifesty ot simvolizma do našich dnej. Hg. Stanislav B. Džimbov. Moskva: Izdatel'skij dom XXI vek - Soglasie, 2000. 147-148.

Burljuk, Nikolaj. „Poetičeskie načala“ [1914]. Literaturnye manifesty ot simvolizma do našich dnej. Hg. Stanislav B. Džimbov. Moskva: Izdatel'skij dom XXI vek - Soglasie, 2000. 149-152.

Dukhan, Igor. „El Lissitzky - Jewish as Universal: From Jewish Style to Pangeometry“. Ars Judaica (2007): 53-72.

El Lisickij: Suprematičeskij skaz pro dva kvadrata. Berlin: Skify [Skythen], 1922.

El Lissitzky. „Kniha z hlediska zrakového dojmu - kniha visuelní“. Typografia 36.8 (1929): 173-180

El Lissitzky. „Typographische Tatsachen“. Gutenberg-Festschrift zur Feier des 25jährigen Bestehens des Gutenbergmuseums in Mainz. Hg. Aloys Ruppel. Mainz: Verlag der GutenbergGesellschaft, 1925. 152-154.

El Lissitzky. „Unser Buch“. Gutenberg-Jahrbuch (1926/27): 172-178, Tafeln XIII-XVI.

Ėrenburg, I'ja. Šest' povestej o legkich koncach. Moskva/Berlin: Gelikon [Helikon], 1922.

Eygns. Hg. Dovid Bergelson, Kiev: Kultur lige, 1920.

Friedman, Mira. „Icon Painting and Russian Popular Art as Sources of some Works by Chagall“. Journal of Jewish Art 5 (1978): 94-110.

Gilbert, Annette. „,Asymmetrische Typographie‘. Zu den Lücken der Schrift in der jüdischen Tradition“. Von Lettern und Lücken. Zur Ordnung der Schrift im Bleisatz. Hg. Mareike Gürtler und Rea Köppel. München: Fink, 2012. 185-205.

Hartog, Arie. „EHYeH ASheR EHYeH. Eine Ausstellung voller Anmerkungen“. Joseph Semah. „Ich bin, der ich bin "EHYeH ASheR EHYeH. Bremen: Gerhard-Marcks-Haus, 2009. 16-27.

Hofštejn, Dovid. Troyer. Kiev: Kultur lige, 1922. 
Hultsch, Anne. „Buchtypographie in den slavischen Avantgarden“. Schrift und Graphisches im Vergleich. Hg. Monika Schmitz-Emans, Linda Simonis und Simone Sauer-Kretschmer. Bielefeld: Aisthesis, 2019. 421-433.

Janecek, Gerald. The Look of Russian Literature. Avant-Garde Visual Experiments, 1900-1930. Princeton: UP, 1984.

Kacis, Leonid. „,'Černyj kvadrat‘ Kazimira Maleviča i ,Skaz pro dva kvadrata“ Ėl'-Lisickogo v iudejskou perspektive“. Leonid Kacis. Russkaja èschatologija i russkaja literatura. Moskva: Ogi, 2000.132-139.

Kacis, Leonid. „Ideologija vitebskogo Unovisa, lerusalimskij Chram i Talmud (Kvadraty K. S. Maleviča i Ėl'-Lisickogo)“. Toronto Slavic Quarterly 12 (spring 2005). http://sites.utoronto.ca/ tsq/12/katzis12.shtml (3. September 2019).

Kacis, Leonid. „,Iudejskaja tajnopis‘ i šifry russkogo avangarda“. Lechaim 7.171 (2006/5766). https://lechaim.ru/ARHIV/171/katsis.htm (27. August 2019). o. P.

Kazovskij, Gillel' (Grigorij). Chudožniki Kul'tur-Ligi. Moskva: Mosty kul'tury, 2002.

Kazovskij, Gilel'/Kazovsky, Hillel. Knižkova grafika mitciv Kul'tur-Ligi. The Book Design of KulturLige Artists. Kiev: Duch i litera, 2011.

Koller, Sabine. „Das Leiden im Angesicht der Kinder - Marc Chagall illustriert Dovid Hofshteyns Gedichtzyklus Troyer (Trauer, 1922)“. Wiener Slawistischer Almanach 64 (2009): 57-94.

Koller, Sabine. „Das Schwarze Quadrat und die jüdische Kunst: Chagall, Lisickij und Malevič in Vitebsk“. Aschkenas 24.1 (2014): 5-31.

Kručenych, Aleksej. Vzorval'. [Sankt Peterburg]: Izd-vo Kuz'mina i Dolinskogo, (2. Aufl.) 1914.

Lisickij, Lazar'. Novaja kul'tura [1919]. http://chagal-vitebsk.com/node/61 (3. September 2019).

Majakovskij, Vladimir. Dlja golosa. Moskva/Berlin: Gosudarstvennoe izdatel'stvo R.S.F.S.R., 1923.

Nisbet, Peter. „El Lissitzky - eine Einführung“. El Lissitzky 1890-1941. Retrospektive. Hannover: Sprengel Museum, 1988a. 10-43.

Nisbet, Peter. „El Lissitzkys typografische Arbeiten. Ein Werkverzeichnis“. El Lissitzky 1890-1941. Retrospektive. Hannover: Sprengel Museum, 1988b. 281-298.

Raskin, Ben Zion. 4 Teyashim. Warszawa: Tarbut, 1922.

Read Full Text. Joseph Semah over El Lissitzky en Barnett Newman. Hg. Joseph Semah und Ulco Mes. Venlo: Museum van Bommel van Dam, 2006.

Rojzman, Matvej. Chevronskoe vino. Moskva: Vserossijskij sojuz poètov, 1923.

Schütz, Christiane. „Kunst aus jüdischen Verlagen“. Europäische Moderne. Buch und Graphik aus Berliner Kunstverlagen 1890-1933. Hg. Lutz S. Malke. Berlin: Reimer, 1989. 141-160.

Šklovskij, Viktor. „,Iskusstvo, kak priem“ / ,Die Kunst als Verfahren““. Texte der russischen Formalisten. Band I. Texte zur allgemeinen Literaturtheorie und zur Theorie der Prosa. Hg. Jurij Striedter. München: Fink, 1969. 2-35.

Valéry, Paul. „Die beiden Tugenden eines Buches“ [1926]. Paul Valéry. 6. Zur Ästhetik und Philosophie der Künste. Frankfurt am Main/Leipzig: Insel-Verl., 1995. 467-471.

Wolitz, Seth L. „The Jewish National Art Renaissance in Russia“. Tradition and Revolution. The Jewish Renaissance in Russian Avant-Garde Art 1912-1928. Hg. Ruth Apter-Gabriel. Jerusalem: Israel Museum, 1987. 21-42.

Wolitz, Seth. „Chagall's last Soviet performance: The graphics for Troyer, 1922“. Jewish Art 1995/ 6: 95-115. 